OPEN ACCESS

Edited by:

Soumitra Paul,

University of Calcutta, India

Reviewed by:

Fangsen $X u$

Huazhong Agricultural University,

China

Ji Sun,

Rockefeller University, USA

*Correspondence:

Jianli Yang

yangjianli@zju.edu.cn;

Wei Fan

fanwei1128@aliyun.com

${ }^{\dagger}$ These authors have contributed equally to this work.

Specialty section: This article was submitted to Plant Nutrition,

a section of the journa Frontiers in Plant Science

Received: 20 February 2016 Accepted: 31 March 2016 Published: 19 April 2016

Citation:

Liu M, Xu J, Lou H, Fan W, Yang J and Zheng S (2016) Characterization of VUMATE1 Expression in Response to Iron Nutrition and Aluminum Stress

Reveals Adaptation of Rice Bean Nigna umbellata) to Acid Soils through Cis Regulation. Front. Plant Sci. 7:511. doi: 10.3389/fpls.2016.00511

\section{Characterization of VUMATE1 Expression in Response to Iron Nutrition and Aluminum Stress Reveals Adaptation of Rice Bean (Vigna umbellata) to Acid Soils through Cis Regulation}

\author{
Meiya Liu',2t, Jiameng Xu' ${ }^{1+}$, Heqiang Lou' ${ }^{1}$, Wei Fan ${ }^{3 *}$, Jianli Yang ${ }^{1 *}$ and Shaojian Zheng ${ }^{1}$ \\ 'State Key Laboratory of Plant Physiology and Biochemistry, College of Life Sciences, Zhejiang University, Hangzhou, China, \\ ${ }^{2}$ Key Laboratory of Tea Biology and Resources Utilization, Ministry of Agriculture, Tea Research Institute, Chinese Academy \\ of Agricultural Sciences, Hangzhou, China, ${ }^{3}$ College of Resources and Environment, Yunnan Agricultural University, \\ Kunming, China
}

Rice bean (Vigna umbellata) VUMATE1 appears to be constitutively expressed at vascular system but root apex, and Al stress extends its expression to root apex. Whether VUMATE1 participates in both Al tolerance and Fe nutrition, and how VUMATE1 expression is regulated is of great interest. In this study, the role of VuMATE1 in Fe nutrition was characterized through in planta complementation assays. The transcriptional regulation of VUMATE1 was investigated through promoter analysis and promoter-GUS reporter assays. The results showed that the expression of VUMATE1 was regulated by Al stress but not Fe status. Complementation of frd3-1 with VUMATE1 under VUMATE1 promoter could not restore phenotype, but restored with 35SCaMV promoter. Immunostaining of VUMATE1 revealed abnormal localization of VUMATE1 in vasculature. In planta GUS reporter assay identified Al-responsive cis-acting elements resided between -1228 and -574 bp. Promoter analysis revealed several cis-acting elements, but transcription is not simply regulated by one of these elements. We demonstrated that cis regulation of VUMATE1 expression is involved in Al tolerance mechanism, while not involved in Fe nutrition. These results reveal the evolution of VUMATE1 expression for better adaptation of rice bean to acid soils where Al stress imposed but Fe deficiency pressure released.

\footnotetext{
Keywords: acid soil, aluminum toxicity, cis-acting element, citrate secretion, evolution, Fe deficiency, transcriptional regulation
}

\section{INTRODUCTION}

Multidrug and toxic compound extrusion (MATE) protein forms a large family of transporters in prokaryotes and eukaryotes where they perform a broad range of functions with diverse substrates (Omote et al., 2006). Some localize to the tonoplast where they transport secondary metabolites into the vacuole. For examples, anthoMATE in Vitis vinifera is an $\mathrm{H}^{+}$-dependent acylated anthocyanin 
transporter (Gomez et al., 2009) and the NtMATE1 and NtMATE2 proteins in Nicotiana tabacum are $\mathrm{H}^{+}$-dependent nicotine transporters (Shoji et al., 2009). EDS5 is a MATE protein in Arabidopsis exporting salicylic acid (SA) from the chloroplast to the cytoplasm (Serrano et al., 2013), while DTX50 functions as an abscisic acid (ABA) efflux transporter (Zhang et al., 2014).

A sub-group of MATE proteins in plants function as citrate transporters and perform at least two separate physiological roles. One role is involved in the translocation of iron (Fe) from the roots to the shoots, such as Ferric Reductase Deficient $\underline{3}$ (FRD3) in Arabidopsis (Durrett et al., 2007) and OsFRDL1 (for FRD3like 1) in rice (Oryza sativa; Yokosho et al., 2009). These proteins are mainly expressed in the pericycle where they transport citrate into the xylem. Knockout mutations of these genes in Arabidopsis and rice result in $\mathrm{Fe}$ accumulation in the root vasculature. The second role for this sub-group of MATE proteins involves in $\mathrm{Al}^{3+}$ tolerance, such as HvAACT1 (Aluminum-activated citrate transporter 1) in barley (Hordeum vulgare; Furukawa et al., 2007) and SbMATE in sorghum (Sorghum bicolor; Magalhaes et al., 2007). These proteins are expressed in the root apices and they facilitate the $\mathrm{Al}^{3+}$-activated secretion of citrate which protects the growing root apices from $\mathrm{Al}^{3+}$ toxicity in acid soils. Therefore, the functions of MATE proteins that transport citrate depend on where they are expressed in the plant. Interestingly, recent evidence from rice and wheat (Triticum aestivum) indicates that a single citrate transporter can perform both physiological roles (Fujii et al., 2012; Tovkach et al., 2013). For instance, in $\mathrm{Al}^{3+}$ sensitive genotypes of barley, HvAACT1 is expressed in the vascular system of the root where it transports citrate into the xylem for efficient translocation of Fe to the shoots. However, in $\mathrm{Al}^{3+}$-tolerant genotypes of barley, a $1 \mathrm{~kb}$ insertion in $5^{\prime}$ UTR region of $H v A A C T 1$ extends expression of this gene to the root apices that results in citrate secretion from these cells as well (Fujii et al., 2012). Similarly, TaMATE1B gene in the wheat is expressed in the vasculature of roots and induced by $\mathrm{Fe}$ deficiency. However, some $\mathrm{Al}^{3+}$-tolerant genotypes of wheat have a $11.1 \mathrm{~kb}$ transposon-like insertion in $25 \mathrm{bp}$ upstream of the start codon of TaMATE1B which constitutively enhances its expression in root apices, increases citrate secretion from these cells and contributes to $\mathrm{Al}^{3+}$ tolerance (Tovkach et al., 2013). Thus, the physiological functions of these proteins have been extended through mutations which alter their tissuespecific expression pattern. These findings are consistent with the hypothesis that the original functions of these genes are altered or extended by mutations to the coding or regulatory regions of the gene (Ryan and Delhaize, 2010).

VuMATE1 from rice bean (Vigna umbellata) is another example of a MATE protein that transports citrate. It was first isolated from $\mathrm{Al}^{3+}$-stressed root tips and mediates $\mathrm{Al}^{3+}$ activated release of citrate from those cells (Yang et al., 2011). On the one hand, VuMATE1 is not expressed in the root apices in the absence of $\mathrm{Al}$ stress, but induced by $\mathrm{Al}$ stress after $3 \mathrm{~h}$ of exposure (Liu et al., 2013). This raises the first question how the expression of VuMATE1 was regulated under Al stress. Gene expression is regulated by different mechansims, such as chromatin condensation, DNA methylation, transcriptional initiaition, alternative splicing of RNA, mRNA stability and so on (Wray et al., 2003). In the case of TaMATE1B and HvAACT1, transposon-like element (TE) insertion in the promoter results in extention of expression into root apices and confers Al tolerancce phenotype. A miniature inverted repeat transposable element (MITE) insertion in the promoter region of SbMATE in sorghum was suggested to be involved in regulating the expression of this gene (Magalhaes et al., 2007). Whether similar mechanism is involved in VUMATE1 expression regulation or not deserves to be investigated. On the other hand, VuMATE1 seems to be constitutively expressed in the vascular system of mature roots cells (Liu et al., 2013). This raises the second question whether VuMATE1 plays roles in Fe nutrition as found for some other members of the family.

In this study, we first evaluated the contribution of VuMATE1 to Fe nutrition using in planta complementation assays, and then explored the role of promoter (cis-regulatory sequences) in transcriptional regulation of VUMATE1 expression in response to Al stress by means of GUS staining assays of VUMATE1 promoter-GUS fusion transgenic lines. The results show that $V U M A T E 1$ is expressed in epidermis and vasculature of mature roots but it is not involved in citrate release from roots of $\mathrm{Fe}$ deficient plants or in the translocation of Fe to the shoots. We further demonstrate that cis-regulation is involved in Al-induced expression of $V U M A T E 1$, and the cis-acting elements involved in root-tip-specific and Al-inducible expression of VUMATE1 resided in promoter region between -1228 and $-574 \mathrm{bp}$. We discuss these results with respect to the adaptation of rice bean to acid soils.

\section{MATERIALS AND METHODS}

\section{Isolation of VuMATE1 Promoter}

The promoter of $V u M A T E 1$ was isolated from genomic libraries that have been constructed before (Liu et al., 2013). Nested PCR was performed using the outer/inner adaptor primer provided by the GenomeWalker ${ }^{\mathrm{TM}}$ Universal Kit (Clontech, Mountain View, CA, USA) and two VUMATE1 gene-specific primers (Supplementary Table S1). The amplified fragments were cloned into the pMD18-T vector (Takara, Dalian, China). The sequences that extends upstream of the cDNA clones were isolated as the $5^{\prime}$-upstream regions of the gene.

\section{Identification of Transcription Start Site}

To determine the transcription start site (TSS) of VuMATE1, total RNA from Al-stressed root apices was isolated with an RNAprep pure Plant Kit (Tiangen, Beijing, China). 5' -RACE was performed using SMART ${ }^{\mathrm{TM}}$ RACE cDNA Amplification Kit (Clontech). Gene-specific primers for 5'-RACE amplification are listed in Supplementary Table S1. Amplified cDNA fragments were cloned into the pMD18-T clone vector (Takara) and sequenced.

\section{Vector Construction and Genetic Transformation}

A series of $5^{\prime}$ deleted promoters, $-1720,-1228,-574$, and -192 bp from the TSS of VuMATE1, were amplified by PCR 
method (Supplementary Figure S1). The primer sequences are shown in Supplementary Table S1. The amplified fragments were cloned into the pMD18-T vector (Takara) and their sequences were confirmed by DNA sequencing. The verified fragments were excised using KpnI and NcoI sites at the $5^{\prime}$ ends of forward and reverse primers, respectively, and inserted at the corresponding sites of pCAMBIA1301. The plasmid containing $-1720 \mathrm{bp}$ construct was also cloned into pCAMBIA1302 at the KpnI and NcoI sites creating an in-frame translational fusion to the GFP gene that was confirmed by DNA sequencing. These constructs was moved into Agrobacterium strain EHA105 and transformed into wild-type Col-0 plants (Clough and Bent, 1998).

For complementation assays, the VUMATE1 coding sequence without stop codon was amplified via PCR method (Supplementary Table S1). The purified fragment was subcoloned into the constructed VuMATE1p::GFP plasmid between VuMATE1p and GFP. The generated plasmid was moved into Agrobacterium strain EHA105 and transformed into frd3-1 homozygous lines.

For the construction of transgenic lines over-expressing VuMATE1, a previous constructed vector harboring 35S::VuMATE1 was used to electroporate into EHA105 (Yang et al., 2011), and transformed into frd3-1.

For all transgenic plant lines, T1 seeds were collected from transgenic T0 plants, and were surface sterilized and planted on one-half-strength Murashige and Skoog medium supplemented with hygromycin. The resistant plants were transferred to soil and allowed to set seeds (T2). Transgenic lines that displayed a 3:1 ratio for hygromycin resistance in the T3 generation were selected for further analysis. All experiments were performed using plants corresponding to the T4 or T5 generation.

\section{Growth Conditions}

For rice bean experiments, rice bean [V. umbellata (Thunb.) Ohwi and Ohashi] seeds were soaked in deionized water overnight, and germinated at $26^{\circ} \mathrm{C}$ in the dark. After germination, the seeds were transferred to a net floating on a $0.5 \mathrm{mM} \mathrm{CaCl}_{2}$ solution ( $\left.\mathrm{pH} 5.5\right)$. The solution was renewed daily. At day 3 after germination, seedlings were transferred to a 1.2-L plastic pot (four holes per pot, two seedlings for each hole) containing aerated one-fifth-strength Hoagland nutrient solution ( $\mathrm{pH}$ 5.5). For Fe-deficiency experiment, plants were cultivated for 1 week in Fe sufficient conditions (1/5 Hoagland solution) and then transferred to the same nutrient solution with or without Fe-EDTA $(20 \mu \mathrm{M})$ for 12 days. The treatment solution was renewed every 3 days.

For Arabidopsis experiments, seeds were surface-sterilized in $75 \%$ ethanol for $4 \mathrm{~min}$, and subsequently rinsed thoroughly with sterile water. Seeds were stratified at $4^{\circ} \mathrm{C}$ for 2 to 4 days before being planted on Petri dishes with 1/5 Hoagland nutrient solution supplemented with $1 \mathrm{mM}$ MES and $0.8 \%$ agar ( $\mathrm{pH} 5.5$ ). After 1 week, the seedlings with uniform size were selected to be transferred onto a net floating on one-fifth-strength Hoagland nutrient solution $(\mathrm{pH}$ 5.5). The solution was renewed every 3 days.

All of the experiments were carried out in an environmentally controlled growth room under $12 \mathrm{~h} 24^{\circ} \mathrm{C}: 12 \mathrm{~h} 22^{\circ} \mathrm{C}$, light : dark, a light intensity of $200 \mu \mathrm{mol}$ photons $\mathrm{m}^{-2} \mathrm{~s}^{-1}$ and $70 \%$ relative humidity.

\section{RT-PCR and qRT-PCR}

Plant materials were ground in liquid nitrogen, and total RNA was extracted using an RNAprep pure Plant Kit (Tiangen). Firststrand cDNAs were synthesized using a PrimeScript ${ }^{\mathrm{TM}}$ RT-PCR Kit (Takara), and diluted to $100 \mathrm{ng} \mu \mathrm{L}^{-1}$. Semi-quantitative RT-PCR was performed with the diluted cDNA as template. Quantitative real-time PCR (qRT-PCR) was performed on a LightCycler480 machine (Roche Diagnostics, Indianapolis, IN, USA) using a SYBR PremixEx Taq kit (Takara). Primer pairs used in both RT- and qRT-PCR were listed in Supplementary Table S1. Three biological replicate RNA/cDNA samples were generated, and each cDNA sample was performed with triplicate technical replicates, from which the relative expression was calculated against that of the internal control gene $18 S$ rRNA using the

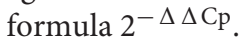

\section{Collection and Analysis of Root Exudates}

After treatments, the roots were briefly washed with $0.5 \mathrm{mM}$ $\mathrm{CaCl}_{2}$ solution ( $\mathrm{pH} 5.5$ ), and then root exudates were collected in $0.5 \mathrm{mM} \mathrm{CaCl}_{2}$ solution $(\mathrm{pH} 4.5)$ either in the absence or presence of $25 \mu \mathrm{M} \mathrm{Al}$ for $6 \mathrm{~h}$ the collected root exudates were purified and concentrated according to Yang et al. (2006). The concentration of citrate was analyzed enzymatically (Delhaize et al., 1993).

\section{Immunostaining of VuMATE1}

The synthetic peptide ATTDNNDIETGDEG-C (positions 173186) was used to immunize rabbits to obtain antibodies against VuMATE1 (Genscript, Nanjing, China). One-week-old seedlings were used to immunolocalization of VuMATE1. The procedure was following previous report (Yamaji and Ma, 2007).

\section{Ferric Chelate Reductase Activity Measurement}

Ferric chelate reductase (FCR) activity was determined according to the previous study (Yang et al., 2013). In brief, the whole excised root (c. $1.0 \mathrm{~g}$ ) was placed in a tube filled with $50-\mathrm{ml}$ of assay solution, which consisted of $0.5 \mathrm{mM} \mathrm{CaSO}_{4}, 0.1 \mathrm{mM}$ MES, $0.1 \mathrm{mM}$ BPDS, and $100 \mu \mathrm{M}$ Fe-EDTA at pH 5.5 adjusted with $1 \mathrm{M} \mathrm{NaOH}$. The tubes were placed in a dark room at $25^{\circ} \mathrm{C}$ for $1 \mathrm{~h}$, with periodic hand-swirling at 10-min intervals. The absorbance of the assay solutions was measured at $535 \mathrm{~nm}$, and the concentration of $\mathrm{Fe}(\mathrm{II})[\mathrm{BPDS}]_{3}$ was quantified using a standard curve.

\section{Chlorophyll Extraction and Quantification}

After 3 weeks culture on hydroponics, newly expanded leaves were harvested. Chlorophyll was extracted in methanol and absorbance measured at 652, 665, and $750 \mathrm{~nm}$. Total chlorophyll concentration was calculated as described (Porra et al., 1989). 


\section{Microscopy}

For GUS histochemical staining, seedlings (10-day-old) were incubated with the substrate 5-bromo-4-chloro-3-indolyl $\beta$-Dglucuronide as described (Jefferson et al., 1987). To localize $\mathrm{Fe}^{3+}$, seedlings after 3 weeks culture were vacuum-infiltrated with Perls stain solution [equal volumes of $4 \%(\mathrm{v} / \mathrm{v}) \mathrm{HCl}$ and $4 \%(\mathrm{w} / \mathrm{v})$ potassium ferrocyanide] for $30 \mathrm{~min}$. Seedlings were then rinsed with water, observed, and photographed with a Nikon AZ100 microscope (Tokyo, Japan). The fluorescence of VuMATE1p::GFP in Arabidopsis was observed with a confocal laser scanning microscope (LSM710: Karl Zeiss, Jena, Germany). For imaging GFP, the $488 \mathrm{~nm}$ line of the Argon laser was used for excitation and emission was detected at $520 \mathrm{~nm}$.

\section{RESULTS}

\section{VuMATE1 Promoter Structure Analysis}

To investigate regulatory mechanisms of VUMATE1 expression, we analyzed the promoter sequence of VUMATE1. We obtained 2-kb DNA sequence upstream of VUMATE1 translation start codon (ATG) (Supplementary Figure S1A). We next determined the potential transcription start site (TSS) of VUMATE1 using $5^{\prime}$-RACE, and only one TSS (A, +1 position) in the VuMATE1 promoter was identified (Supplementary Figure S1B). The TSS was located in the $3^{\prime}$ region of the putative TATA box (TATAA, $-35 /-30 \mathrm{bp})$. There is an intron of $170 \mathrm{bp}$ in length between TSS and translation start codon (Supplementary Figure S1A).

\section{Expression Patterns of VuMATE1 under Fe Deficiency and AI Stress}

We have previously demonstrated that the expression of VUMATE1 is induced by $\mathrm{Al}$ stress in root tip region (Liu et al., 2013). However, tissue expression localization of VuMATE1 in transgenic Arabidopsis plants carrying $\beta$-glucuronidase (GUS) reporter gene under control of VUMATE1 promoter showed that VUMATE1 is constitutively expressed at vasculature of maturation root zone (Liu et al., 2013). Here, we further examined the expression and localization of VUMATE1 in vivo. We transformed wild-type Arabidopsis (col-0) with a construct that harbors the VUMATE1 promoter upstream of green fluorescent protein (VuMATE1p::GFP). GFP signal was not observed in root apex under normal growth conditions, but detectable in maturation root zone (Supplementary Figure S2A). Detailed analysis of the expression of VuMATE1p::GFP revealed that it was mainly expressed in epidermis and vasculature of maturation root zone (Supplementary Figures S2C,D). Exposure of roots to $\mathrm{Al}$ for $9 \mathrm{~h}$ resulted in significant increase of VuMATE1p::GFP expression in root apex (Supplementary Figure S2B), but had no effects in maturation root zone (Supplementary Figure S2D).

To examine whether the expression of VUMATE1 is regulated by Fe status, we analyzed the response of VUMATE1 expression to Fe nutrition by RT- and qRT-PCR. After 12 days culture of rice bean seedlings in nutrient solution with $(+\mathrm{Fe})$ or without $\mathrm{Fe}(-\mathrm{Fe})$, newly expanded leaves of $-\mathrm{Fe}$ plants displayed obvious chlorosis symptom (Supplementary Figure S3). SPAD values (indications of chlorophyll content) of newly expanded leaves decreased by $\sim 60 \%$ following this treatment compared to $+\mathrm{Fe}$ conditions (Figure 1A) and the ferric chelate reductase (FCR) activity in roots increased by almost threefold (Figure 1B), demonstrating that the plants were Fe-deficient after 12 days treatment. This is supported by RT-PCR and qRT-PCR analysis indicating that expression of VuIRT1 (Iron Regulated Transporter 1), the ferrous Fe transporter, was fifteen times greater in the roots of $-\mathrm{Fe}$ plants compared to $+\mathrm{Fe}$ controls (Figures 1C,D). By contrast, VUMATE1 expression in roots was unaffected by Fe status since expression levels were similar in the $-\mathrm{Fe}$ and $+\mathrm{Fe}$ plants (Figure 1E).

\section{VuMATE1 cannot Complement the frd3-1 Phenotype}

The localization of VUMATE1 to the vascular system prompted us to investigate whether VUMATE1 is also involved in Fe nutrition similar to HvAACT1 in barley (Fujii et al., 2012) and TaMATE1B in wheat (Tovkach et al., 2013). We expressed VUMATE1 using its native promoter in the Arabidopsis mutant frd3-1 (VuMATE1p::VuMATE1/frd3-1), which is defective in Fe translocation (Green and Rogers, 2004), and two independent transgenic lines (line1 and line2) were used for further analysis. RT-PCR analysis indicated that VUMATE1 was expressed in both transgenic lines but not in frd3-1 mutant (Figure 2A). When grown in one-fifth-strength Hoagland nutrient solution, newly expanded leaves of the frd3-1 mutant lines exhibited severe chlorosis (Figure 2B), which is in accordance with lower chlorophyll levels (Figure 2C). Perls blue staining demonstrated that significantly more $\mathrm{Fe}$ was accumulated in the root vasculature of $f r d 3-1$ than WT plants (Supplementary Figure S4), which is consistent with previous descriptions of this mutant (Durrett et al., 2007). The frd3-1 lines transformed with VuMATE1p::VUMATE1 showed these same general symptoms (Figure 2B). Chlorophyll content of the newly expanded leaves in frd3-1 and the two independent complementation lines was approximately half of WT levels (Figure 2C). Perls blue staining of roots showed similarly high Fe precipitation in the vasculature of the complementation lines (Supplementary Figure S4), indicating that $\mathrm{Fe}$ translocation to the shoots was reduced in all the lines. These results indicate that VuMATE1 expression driven by its native promoter could not complement the mutant phenotype of frd3-1.

We have previously demonstrated that VuMATE1 is a plasma membrane-localized citrate-permeable transporter protein (Yang et al., 2011). Thus, the inability of VUMATE1 to restore frd31 phenotype with respect to $\mathrm{Fe}$ nutrition suggests that the expression pattern but not gene function is responsible for the loss of its role in $\mathrm{Fe}$ nutrition. To test this hypothesis, we introduced VuMATE1 using 35S CaMV promoter into the frd3-1 mutant (35S::VuMATE1/frd3-1). The chlorosis was greatly, albeit not completely, restored in two independent transgenic lines, OX1 and OX2 (Figure 3A). In addition, Perls blue staining result also showed that the accumulation of $\mathrm{Fe}$ in root vasculature 
A

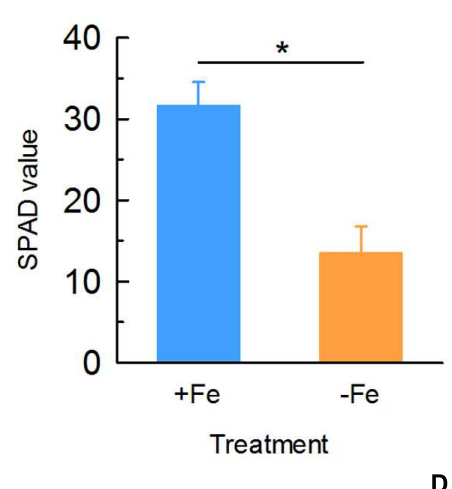

C

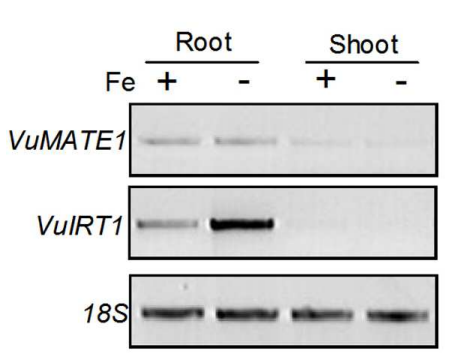

B

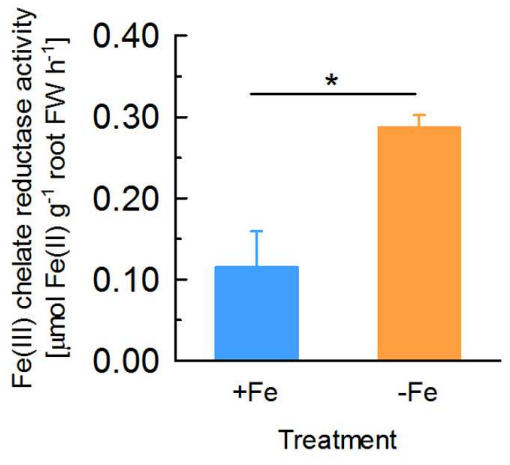

E
D

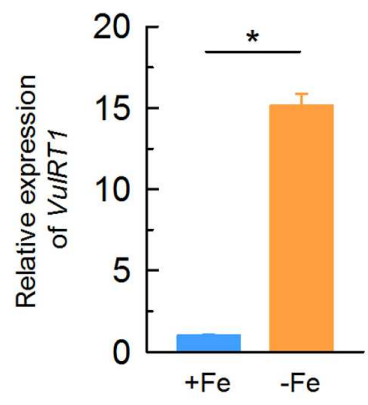

Treatment

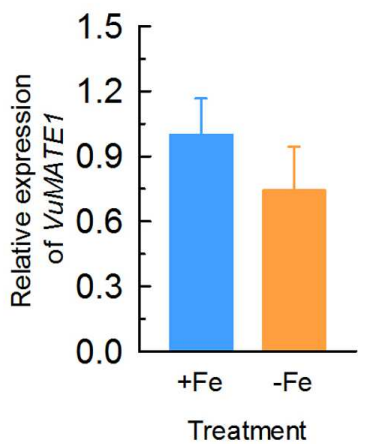

FIGURE 1 | Physiological and molecular responses of rice bean to Fe deficiency. Seedlings of rice bean were subject to nutrient solution with $20 \mu \mathrm{M}$ Fe $(+\mathrm{Fe})$ or without $(-\mathrm{Fe})$ for 12 days. (A) SPAD value of newly expanded leaves. Data are means $\pm \mathrm{SD}(n=8)$. (B) Ferric chelate reductase (FCR) activity of roots. Data are means $\pm S D(n=4)$. (C) RT-PCR analysis of the expression of VuMATE1 and VuIRT1 in both roots and shoots. (D) Quantitative real-time PCR analysis of the expression of VuIRT1 in roots. Data are means \pm SD $(n=3)$. (E) Quantitative real-time PCR analysis of the expression of VuMATE1 in roots. Data are means \pm SD $(n=3)$. Asterisks represent significant differences between $+\mathrm{Fe}$ and $-\mathrm{Fe}$ treatment at $P<0.05$.

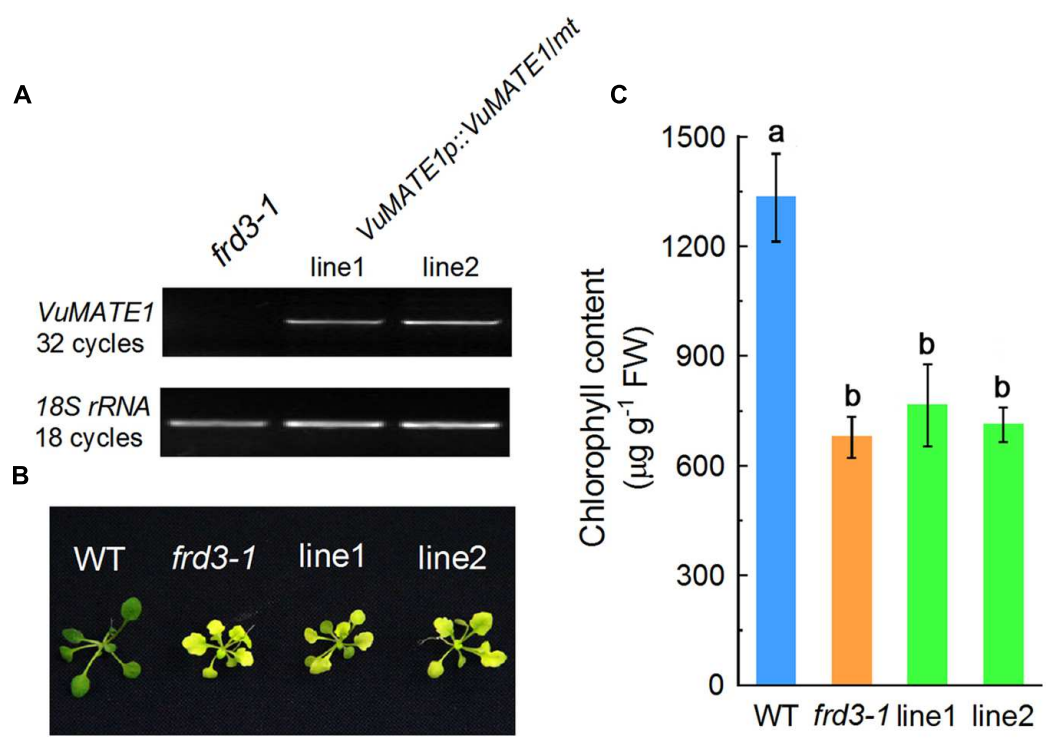

FIGURE 2 | In planta complementation assay of VuMATE1 in Fe nutrition in Arabidopsis mutant frd3-1 driven by its native promoter (VuMATE1p::VuMATE1/frd3-1). (A) RT-PCR characterization of VUMATE1 expression in roots of two independent complemented lines (line 1 and line 2).

(B) Phenotype analysis of leaf chlorosis in wild-type (WT), frd3-1, and two complemented lines. (C) Chlorophyll content of newly expanded leaves in wild-type (WT), frd3-1, and two complemented lines. Data are expressed as means \pm SD $(n=4)$. Columns with different letters are significantly different at $P<0.05$. 


\section{A}

B
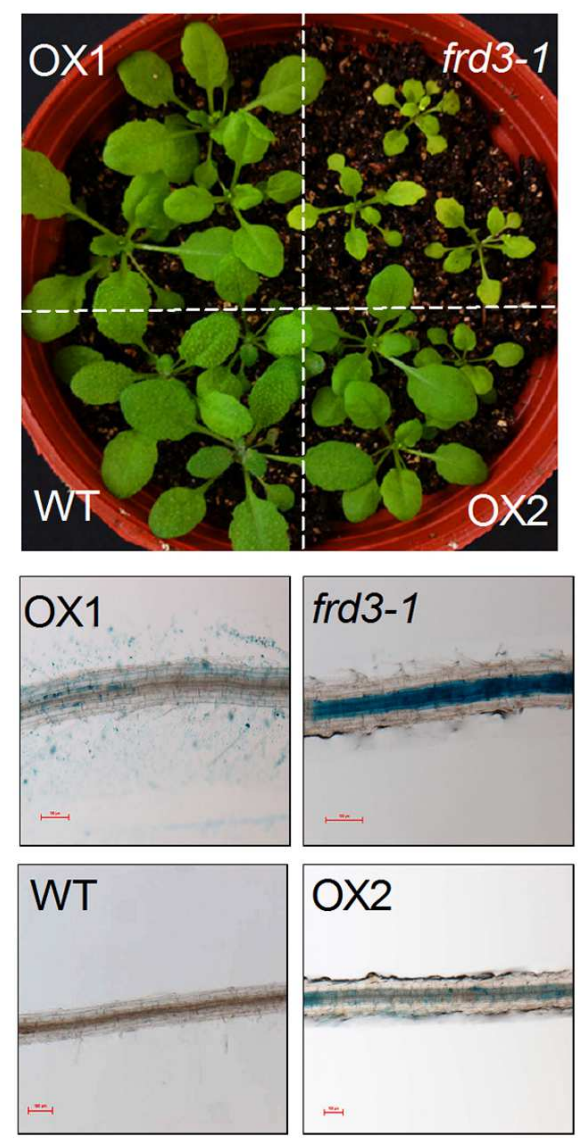

FIGURE 3 | In planta complementation assay of VUMATE1 in Fe nutrition in Arabidopsis mutant frd3-1 driven by 35S CaMV promoter. (A) Phenotype analysis of leaf chlorosis in WT, frd3-1, and two transgenic lines overexpressing VUMATE1 (OX1 and OX2). (B) Root ferric precipitation of WT, frd3-1, and two transgenic lines overexpressing VUMATE1 (OX1 and OX2). Bar, $100 \mu \mathrm{m}$.

was decreased dramatically in comparison to frd3-1 mutant (Figure 3B).

\section{Cell-Specificity of Localization of VuMATE1}

To further investigate why VuMATE1 expression under the control of its native promoter could not complement the frd3-1 mutant, we examined the cell-specificity of localization of VuMATE1 with a polyclonal antibody. In root apex, no fluorescent signal was observed (Figure 4A). However, fluorescence signal was detected in the epidermis of root apex after $9 \mathrm{~h}$ exposure to $25 \mu \mathrm{M} \mathrm{Al}$ (Figure 4B). Moreover, VUMATE1 is localized on the plasma membrane of the distal side of epidermis cell (Figure 4B). Being mainly localized to cells near xylem vessels, and the epidermis in maturation root zone, VuMATE1 could not be detected in the pericycle or in cells internal to the pericycle (Figure 4C).

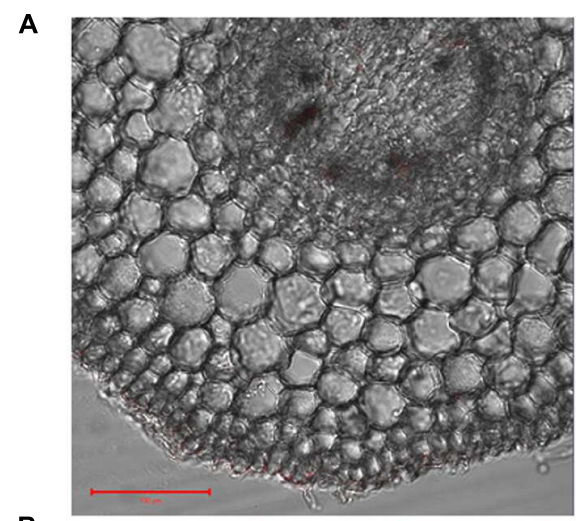

B

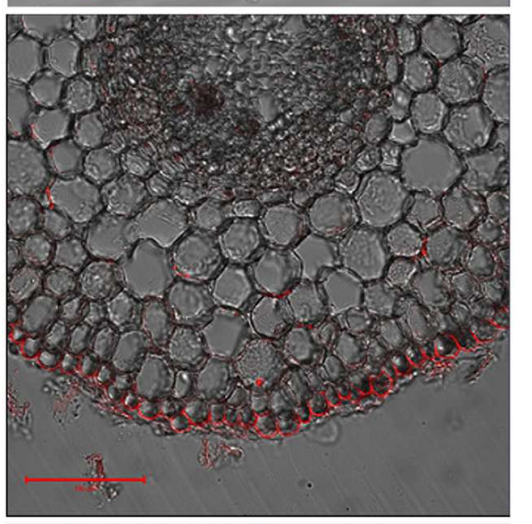

C

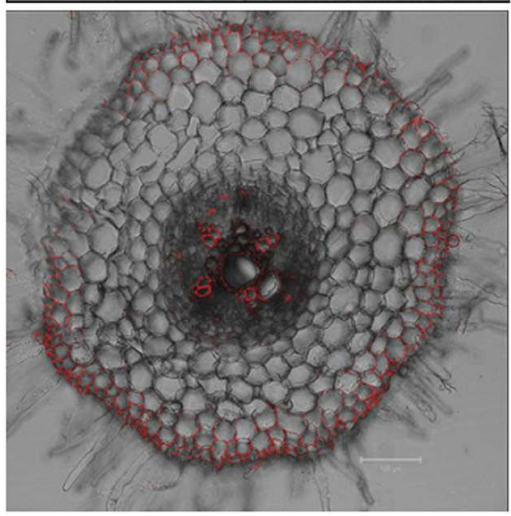

FIGURE 4 | Cell-specificity of localization of VuMATE1 in roots. Immunostaining with anti-VUMATE1 antibody is shown in the root apex either before (A) or after Al stress for $9 \mathrm{~h}$ (B), and maturation root zone (C). Bar, $100 \mu \mathrm{m}$.

\section{Effect of Fe Supply on Citrate Secretion}

Citrate anion secretion from roots can also complex with $\mathrm{Fe}$ in the rhizosphere to improve its mobilization and uptake by roots (Jones et al., 1996). The localization of VuMATE1 in the epidermis cells of the root led us to investigate its possible role in mobilizing $\mathrm{Fe}$ in the rhizosphere through enhanced citrate secretion. Therefore, we analyzed citrate secretion from whole roots under either $+\mathrm{Fe}$ or $-\mathrm{Fe}$ conditions and found that $\mathrm{Fe}$ deficiency did not increase citrate secretion. However, $\mathrm{Al}$ treatment significantly increased citrate secretion regardless of $\mathrm{Fe}$ supply (Figure 5). 


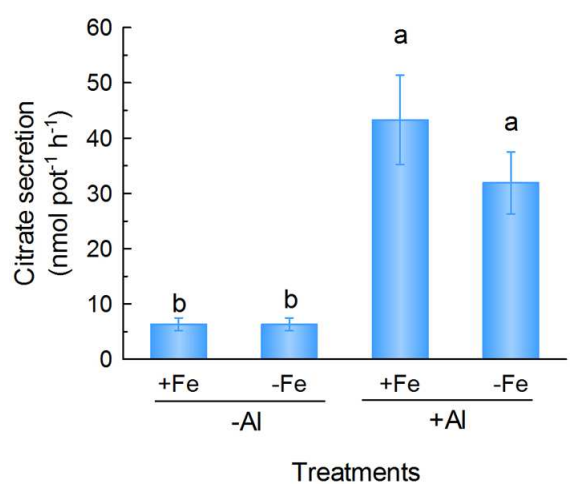

FIGURE 5 | Citrate secretion from rice bean roots in response to Fe deficiency and Al stress. One-week-old seedlings were subject to nutrient solution with $20 \mu \mathrm{M} \mathrm{Fe}(+\mathrm{Fe})$ or without $(-\mathrm{Fe})$ for 12 days after treatment, root exudates were collected in $0.5 \mathrm{mM} \mathrm{CaCl}_{2}$ solution $(\mathrm{pH} 4.5)$ in the absence (-Al) or presence of $25 \mu \mathrm{M} \mathrm{Al}(+\mathrm{Al})$ for $6 \mathrm{~h}$. Data are expressed as means $\pm \mathrm{SD}(n=4)$. Columns with different letters indicate significant difference at $P<0.05$.

\section{Promoter Analysis to Characterize VUMATE1 Expression}

To characterize in detail the regulation of VUMATE1 transcription by $\mathrm{Al}$ stress and $\mathrm{Fe}$ status, we analyzed GUS expression in VuMATE1p::GUS transgenic lines carrying different lengths of the $5^{\prime}$ region of the VUMATE1 promoter. In the absence of Al stress, GUS staining was not observed in the root apex of all promoter-GUS reporter transgenic lines carrying different $5^{\prime}$ deletion promoters (Figure 6A). However, after 9-h exposure to $\mathrm{Al}$ stress, promoter-GUS reporter lines, -1720 and $-1228 \mathrm{bp}:: G U S$, exhibited Al-inducible and root apex staining of GUS activity, whereas GUS staining was not observed in -574 and -192 bp::GUS transgenic lines (Figure 6A). This result indicated that $c i s$-acting elements responsible for root-tip specific and $\mathrm{Al}$-inducible expression resided in VUMATE1 promoter sequence between -1228 and $-574 \mathrm{bp}$. On the other hand, all promoter-GUS reporter transgenic lines examined showed constitutive expression in maturation root zone, even in the shortest -192 bp::GUS transgenic line (Figure 6B).

To find cis-acting elements in the promoter, we first searched the VUMATE1 promoter sequence between -1228 and $-574 \mathrm{bp}$ using the PLACE and PlantCARE databases (Higo et al., 1999; Lescot et al., 2002). As shown in Table 1, both databases predicted cis-acting elements related to drought-inducible element (MBS), ABA response element (ABRE), wounding and pathogen responsive element ( $\mathrm{W}$ box), and SA response element (TCA). We then searched three reported Al-responsive cis-acting elements, i.e., $\mathrm{GGN}(\mathrm{T} / \mathrm{g} / \mathrm{a} / \mathrm{C}) \mathrm{V}(\mathrm{C} / \mathrm{A} / \mathrm{g}) \mathrm{S}(\mathrm{C} / \mathrm{G}) \mathrm{T}$ of $\mathrm{STOP} 1$ ortholog, ART1 (Tsutsui et al., 2011), GGCCCA(T/A) of ASR5 (Arenhart et al., 2014), and CGCG box of CALMODULINBINDING TRANSCRITPION ACTIVATOR (CAMTA; Tokizawa et al., 2015). Only a single cis-acting element of ART1 was found to be located at $-629 \mathrm{bp}$, whereas none of both ASR5 and CAMTA was observed (Table 1).
A
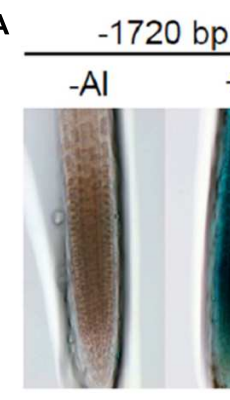

$-A l$ + Al

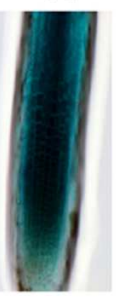

B

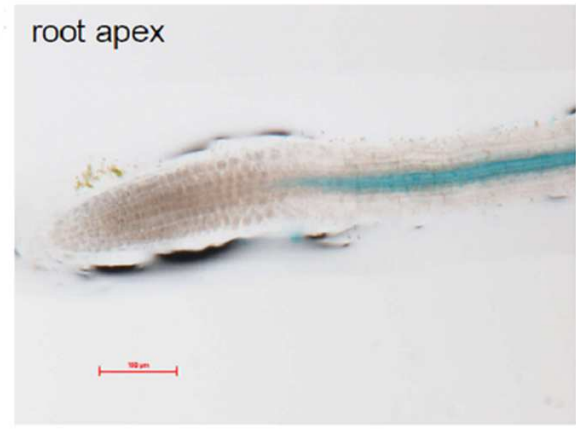

$-574 \mathrm{bp}$

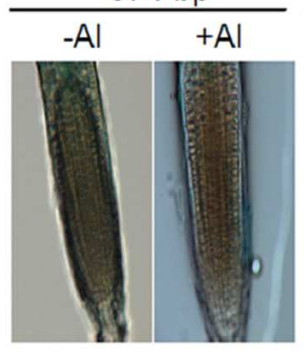

mature root zone

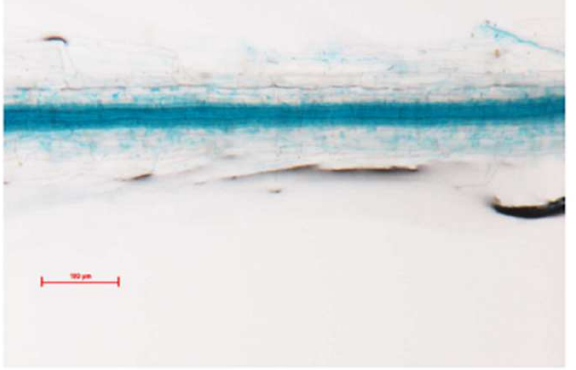

FIGURE 6 | Histochemical analysis of GUS expression in transgenic Arabidopsis lines driven by different $\mathbf{5}^{\prime}$ deletion sequence of VuMATE1 promoter. (A) Induction of VUMATE1 by Al in transgenic Arabidopsis carrying VuMATE1p::GUS constructs. The representative images show GUS staining in roots after 9 h of exposure to $0(-\mathrm{Al})$ or $1 \mu \mathrm{M} \mathrm{Al}(+\mathrm{Al})$ activity in nutrient solution. (B) GUS expression in root apex and maturation root zone of transgenic Arabidopsis carrying the shortest promoter of VUMATE1 (-192 bp::GUS). At least two independent transgenic lines were used to analyze GUS expression. Bar, $100 \mu \mathrm{m}$. 
TABLE 1 | The list of cis-acting elements of the VuMATE1 promoter sequence between -1228 and $-574 \mathrm{bp}$.

\begin{tabular}{|c|c|c|c|c|}
\hline cis-acting element & Motif & Position & Organism & Function \\
\hline ABRE & TGCACGTAT & -610 & Arabidopsis thaliana & cis-acting element involved in the abscisic acid (ABA) responsiveness \\
\hline \multirow[t]{2}{*}{ MBS } & CGGTCA & -808 & Zea mays & MYB binding site involved in drought-inducibility \\
\hline & CAACTG & -586 & Arabidopsis thaliana & \\
\hline W box & TTGACC & -1009 & Arabidopsis thaliana & Elicitation; wounding and pathogen responsiveness. Binds WRKY type transcription factors \\
\hline TCA & TGTTCTTCTC & -1224 & Brassica oleracea & Salicylic acid response element \\
\hline ART1 & GGNVST & -629 & Oryza sativa & Al stress responsiveness \\
\hline
\end{tabular}

In order to examine whether phytohormones, ABA and SA, are involved in the transcriptional regulation of VUMATE1, we compared GUS activities among different treatments, i.e., ABA (10 $\mu \mathrm{M}$; pH 5.5), SA (10 $\mu \mathrm{M}$; pH 5.5), or Al (10 $\mu \mathrm{M} ; \mathrm{pH}$ 5.5). Previous study showed that apart from Al, low $\mathrm{pH}$ (5.0) also affect VUMATE1 expression in GUS transgenic lines (Fan et al., 2015). Therefore, in order to clarify the regulatory role of hormones, we used the pH5.5 as normal growth conditions to eliminate the impact of $\mathrm{pH}$. As shown in Figure 7, under normal growth conditions ( $\mathrm{pH}$ 5.5), GUS activity was undetectable in the entire root apex region. Both $\mathrm{ABA}$ and SA could slightly induce GUS activity restricted to elongation zone. Addition of $\mathrm{Al}$ resulted in the strong induction of GUS activity at entire root apex region.

\section{DISCUSSION}

In the present study, we demonstrated that cis regulation is involved in the transcriptional regulation of VuMATE1 expression in adaptation to acid soils. In planta GUS expression analysis indicated that cis-acting motifs responsible for Alinducible expression reside between -1228 and -574 bp of VUMATE1 promoter (Figure 6). Several lines of evidence suggest

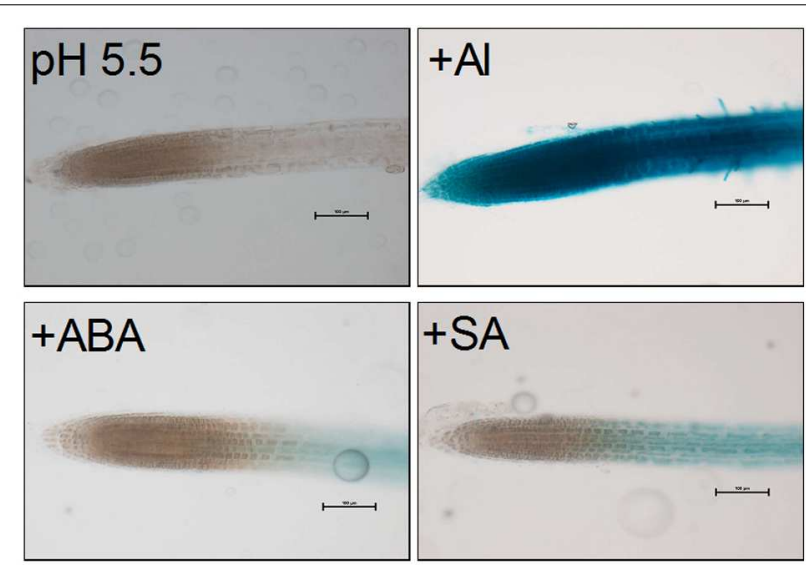

FIGURE 7 | VuMATE1p::GUS expression analysis in transgenic Arabidopsis plants. Transgenic seedlings were exposed to $10 \mu \mathrm{M} \mathrm{Al}, 10 \mu \mathrm{M}$ ABA, or $10 \mu \mathrm{M}$ SA for $9 \mathrm{~h}$. Activation of the VUMATE1 promoter was observed by GUS staining (blue). At least two independent transgenic lines were used to analyze GUS expression. Bars, $100 \mu \mathrm{m}$. that the acquisition of $\mathrm{Al}$ tolerance mechanism can occur through mutations that modify the expression patterns of a single gene. For example, in wheat, the number of tandemly repeated elements of 33-803 bp in length located upstream of TaALMT1 coding region was associated with higher levels of TaALMT1 expression (Sasaki et al., 2006; Ryan et al., 2010). Interestingly, these tandemly repeated elements are absent in the AetALMT1 which is the ortholog of TaALMT1 from Aegilops tauschii, the donor of D genome in hexaploid wheat (Ryan et al., 2010). It was speculated that these tandemly-repeated elements provided some advantage to hexaploid wheat as individuals spread to more acid soils. In Al-tolerant genotypes of barley, a 1-kb insertion upstream of the $H v A A C T 1$ coding region extends the expression of this gene into the root apex (Fujii et al., 2012). Similarly the higher expression level of TaMATE1B in several Brazilian wheat lines was found to be associated with the presence of a Sukkula-like transposable element in its promoter region as well (Tovkach et al., 2013). Apparently, the acquisition of Al tolerance mechanism in rice bean is different from these species, since no insertion segments were observed in the VUMATE1 promoter.

Recently, adaptation of Holcus lanatus to acid soils was achieved by increasing the number of cis-acting elements for the transcription factor, ART1, in the promoter of HIALMT1 (Chen et al., 2013). The expression of Arabidopsis AtALMT1 is also largely attributed to the interaction between AtSTOP1 and its cis-acting element. However, the cis-acting elements and trans-regulatory factors involved in VuMATE1 expression seem to be different from these genes. We have previously demonstrated that an inducible $\mathrm{C}_{2} \mathrm{H}_{2}$-type zinc finger transcription factor, VuSTOP1, can bind to VuMATE1 promoter region between -1228 and $-920 \mathrm{bp}$, but not to the region between -820 and -555 bp where a putative cisacting motif of ART1 resides (Table 1; Fan et al., 2015). Furthermore, in planta complementation of Atstop1 mutant with VUSTOP1 demonstrated that the expression of AtMATE was only slightly restored in the complemented lines (Fan et al., 2015). Thus, it is very likely that transcription factors other than VuSTOP1 are involved in the transcriptional regulation of VUMATE1 under Al stress. Recently, a series of cisacting elements and transcription factors responsible for the expression regulation of AtALMT1 were identified by integrating bioinformatics and molecular biological approaches (Tokizawa et al., 2015). Thus, it is possible that multiple mechanisms are also involved in VUMATE1 expression regulation, which needs further investigation. 
We identified a putative W-box cis-acting motif (TTGACC) in the position of -1009 bp of VUMATE1 promoter (Table 1), which is potentially responsible for the interaction with WRKYtype transcription factors. Recently, Ding et al. (2013) reported that WRKY46 functions as a repressor of AtALMT1, whereas WRKY46 itself is repressive to Al stress. Given that WRKYtype transcription factors were involved in the transcriptional regulation of VUMATE1 expression in rice bean, they will perform as a transcriptional activator instead of repressor. This is evidenced by GUS expression in VuMATE1p::GUS transgenic lines carrying different lengths of the $5^{\prime}$ region of the VuMATE1 promoter. In both -579 and -194 bp::GUS lines, GUS activity was undetectable either in the absence or presence of $\mathrm{Al}$ stress (Figure 6A), indicating it is not repressors but activators to be mainly involved in VUMATE1 expression. Furthermore, pretreatment with $\mathrm{CHX}$, a protein translation inhibitor, resulted in the significantly inhibition of VuMATE1 expression (Liu et al., 2013; Fan et al., 2015), confirming that repressor is not involved in the transcriptional regulation of VUMATE1 expression in response to $\mathrm{Al}$ stress.

Phytohormones are important signal inducers that participate extensively in plant response to environmental stresses (Sun et al., 2010; Tian et al., 2014; Yang et al., 2014). In soybean, $\mathrm{ABA}$ was reported to enhance citrate secretion both in the absence and presence of Al stress (Shen et al., 2004). Al stress resulted in the accumulation of $\mathrm{SA}$, and exogenously application of SA could in turn improve Al tolerance through modulation of citrate secretion in Cassia tora (Yang et al., 2003). In this present study, we identified both potential ABA-responsive and SA-responsive cis-acting elements in the promoter of VuMATE1 (Table 1), suggesting possible involvement of these phytohormones signaling in Al-regulated VuMATE1 expression. However, we found that phytohormones (both ABA and SA) are not the major factors responsible for Al-induced VuMATE1 expression in rice bean root tip, although they induced GUS activity. In planta GUS expression analysis revealed that there were differences in the intensity and tissue localization of the GUS staining between phytohormones (ABA and SA) and Al treatments (Figure 7). Recently, it was demonstrated that several signal inducers, especially IAA and ABA, can trigger AtALMT1 expression in Arabidopsis (Kobayashi et al., 2013). However, the induction of AtALMT1 expression in response to Al stress is independent of IAA and ABA signaling, which in combination with our present observation provides evidence that Al-induced organic acid secretion seems to be independent of signaling from phytohormones.

We found that in control conditions ( $-\mathrm{Al}$ ) the expression of VUMATE1 was absent in the root apex, but constitutively expressed in root hairs, epidermis and vasculature of mature roots (Supplementary Figure S2; Figure 6). This pattern of expression suggested that VuMATE1 could be involved in aspects of Fe nutrition in rice bean. This is based on previous reports that citrate secretion from roots is associated with Fe mobilization from the rhizosphere in chickpea (Cicer arietinum; Ohwaki and Sugahara, 1997) and maize (Zea mays) (Carvalhais et al., 2011). Furthermore, other citrate-transporting MATE proteins such as HvAACT1 in barley (Fujii et al., 2012), TaMATE1B in wheat (Tovkach et al., 2013), FRD3 in Arabidopsis (Rogers and Guerinot, 2002) are induced by Fe deficiency and likely responsible for $\mathrm{Fe}$ translocation to the shoots by loading citrate into xylem. However, here we demonstrated that VuMATE1 was not involved in Fe nutrition in rice bean. This conclusion is supported by the following pieces of evidence.

First, VUMATE1 expression was not affected by Fe deficiency (Figure 1E). There are two potential reasons responsible for the inability of responsiveness of VuMATE1 expression to $\mathrm{Fe}$ nutritional status. One is that there are no Fe-deficiencyresponsive components regulating VUMATE1 expression in rice bean. However, since both VuIRT1 expression and FCR activity was induced by Fe deficiency (Figures 1B,D), Fe-deficiencyresponses are clearly operating in rice bean. The other reason is that there are either no Fe-deficiency-inducible cis-elements in the promoter of VuMATE1, or that such cis-elements were originally present but have been lost or mutated during the adaptation of rice bean to acid soils. Acid soils typically have toxic concentrations of $\mathrm{Al}$ but they rarely induce Fe deficiency in plants because the low $\mathrm{pH}$ can maintain a higher concentration of soluble Fe. Therefore there is less requirements to mobilize Fe from acid soil through the secretion of organic anions. In line with this result, there is only a trace amount of citrate secreted into growth medium and the secretion rate was unaffected by the onset of Fe deficiency (Figure 5). Thus, it is possible that as these plants adapted to acid soils they lost the ciselements responsible for iron-deficiency-inducible expression of VUMATE1 but maintained the induction of expression by Al. Such a change could confer an advantage to rice bean because in addition to improving Al tolerance, it would minimize unnecessary carbon loss in conditions when it would not be beneficial. This is supported by the finding that transgenic tomato lines that express VuMATE1 under control of constitutive 35S CaMV promoter exhibited constitutive citrate efflux (Yang et al., 2011).

The second reason for concluding that VuMATE1 is not involved in Fe nutrition is that VuMATE1 does not appear to translocate Fe from the roots to the shoots. This was supported by the finding that transgenic Arabidopsis lines expressing VUMATE1 under the control of its native promoter could not rescue the phenotype of $f r d 3-1$ mutant which is defective in $\mathrm{Fe}$ translocation (Figure 2). There are two possible reasons for the ineffectiveness of VuMATE1 in Fe translocation. One is that the expression level of VUMATE1 was too low to exhibit a sufficient phenotype (Figure 1C). However, FRD3 expression in wild-type Arabidopsis is also at the limits of detection yet this gene was able to complement the frd3-1 mutant (Green and Rogers, 2004). Thus, the low level of VUMATE1 expression may not be the main reason for its inability to translocate Fe. An alternative possibility is that the tissue-specific localization of VuMATE1 is unsuited for Fe translocation. Both FRD3 in Arabidopsis and OsFRDL1 in rice have been demonstrated to be localized mainly in the pericycle (Green and Rogers, 2004; Yokosho et al., 2009). However, the expression of VuMATE1 was absent in pericycle and cells immediately internal to pericycle (Figure 4). Although it is still not clear how critical localization to the pericycle is for $\mathrm{Fe}$ translocation, the restoration of phenotype by VUMATE1 under 
35S CaMV promoter (Figure 3) suggests that this difference in localization contribute to the ineffectiveness of VuMATE1 in helping Fe move to the shoots.

Why then does VuMATE1 appear to lack a role in Fe nutrition whereas other citrate transporters from the MATE appear to be involved in Fe nutrition as well as Al tolerance? The explanation might be found in the very different origins of rice bean compared to barley and wheat. For example, barley and wheat arose and were originally cultivated in regions with calcareous soils where Fe deficiency is a major constraint to growth (von Bothmer et al., 2003; Ryan et al., 2010). Therefore, both species evolved efficient mechanisms for accessing and taking up Fe from these soils. The adaptation of these species to more acidic soil may have been long enough to evolve $\mathrm{Al}$ tolerance but not to lose their efficient mechanisms for acquiring Fe. Indeed, a recent report concluded that selection pressure for greater $\mathrm{Al}$ tolerance in natural populations of $H$. lanatus required only 150 years for $\mathrm{Al}$ tolerant alleles of HLAMT1 to become more frequent in the surviving population (Chen et al., 2013). By contrast, rice bean was originally cultivated on acid soils where $\mathrm{Al}$ toxicity poses a stronger selection pressure than Fe deficiency (Yang et al., 2006). We hypothesized that the Fe-deficiency-responsive cis-acting element does not evolve for VUMATE1 or such element has lost during long adaptation process to acid soils. The GUS reporter transgenic line carrying the shortest promoter exhibited the same expression patterns to others carrying longer promoter in term of Fe nutrition status provided the circumstantial evidence to support our hypothesis (Figure 6B). However, the evolution of root tip-specific and Alinducible cis-elements in rice bean not only alleviates $\mathrm{Al}$ toxicity but also prevents excessive loss of fixed carbon through citrate secretion.

In summary, we have characterized the expression of VUMATE1 in response to Fe deficiency. We conclude that this gene is not involved with citrate secretion from roots during Fe deficiency and nor is it involved with Fe translocation from

\section{REFERENCES}

Arenhart, R. A., Bai, Y., de Oliveira, L. F., Neto, L. B., Schunemann, M., Maraschin Fdos, S., et al. (2014). New insights into aluminum tolerance in rice: the ASR5 protein binds the STAR1 promoter and other aluminum-responsive genes. Mol. Plant. 7, 709-721. doi: 10.1093/mp/sst160

Carvalhais, L. C., Dennis, P. G., Fedoseyenko, D., Hajirezaei, M. R., Borriss, R., and von Wirén, N. (2011). Root exudation of sugars, amino acids, and organic acids by maize as affected by nitrogen, phosphorus, potassium, and iron deficiency. J. Plant Nut. Soil Sci. 174, 3-11. doi: 10.1002/jpln.201000085

Chen, Z. C., Yokosho, K., Kashino, M., Zhao, F. J., Yamaji, N., and Ma, J. F. (2013). Adaptation to acidic soil is achieved by increased numbers of cis-acting elements regulating ALMT1 expression in Holcus lanatus. Plant J. 76, 10-23. doi: $10.1111 /$ tpj. 12266

Clough, S. J., and Bent, A. F. (1998). Floral dip: a simplified method for Agrobacterium-mediated transformation of Arabidopsis thaliana. Plant J. 16, 735-743. doi: 10.1046/j.1365-313x.1998.00343.x

Delhaize, E., Ryan, P. R., and Randall, P. J. (1993). Aluminum tolerance in wheat (Triticum aestivum L.) II. Aluminum-stimulated excretion of malic acid from root apices. Plant Physiol. 103, 695-702.

Ding, Z. J., Yan, J. Y., Xu, X. Y., Li, G. X., and Zheng, S. J. (2013). WRKY46 functions as a transcriptional repressor of $A L M T 1$, regulating aluminum-induced malate secretion in Arabidopsis. Plant J. 76, 825-835. doi: 10.1111/tpj.12337 the roots to the shoots. The role of VuMATE1 in Al tolerance is controlled by elements in the promoter which respond to $\mathrm{Al}$ stress, via unknown pathways, to increase expression in root apices. The loss or gain of specific physiological functions in response to environmental selection pressures can occur via cis mutations that modify the level and distribution of gene expression.

\section{AUTHOR CONTRIBUTIONS}

JY and SZ conceived the study. ML, JX, HL, and WF performed the experiments and carried out the analysis. ML and JY designed the experiments and wrote the manuscript. All authors read and approved the final manuscript.

\section{FUNDING}

This work was supported by the 973 Project (2014CB441002), the Natural Science Foundation of China (31071849, 31222049, and 31501827), and the Fundamental Research Funds for the Central Universities.

\section{ACKNOWLEDGMENT}

Thanks are given to Dr. Peter R. Ryan (Commonwealth Scientific and Industrial Research Organization Plant Industry, Australia) for his critical reading of the manuscript.

\section{SUPPLEMENTARY MATERIAL}

The Supplementary Material for this article can be found online at: http://journal.frontiersin.org/article/10.3389/fpls.2016.00511

Durrett, T. P., Gassmann, W., and Rogers, E. E. (2007). The FRD3-mediated efflux of citrate into the root vasculature is necessary for efficient iron translocation. Plant Physiol. 144, 197-205. doi: 10.1104/pp.107.097162

Fan, W., Lou, H. Q., Gong, Y. L., Liu, M. Y., Cao, M. J., Liu, Y., et al. (2015). Characterization of an inducible $\mathrm{C} 2 \mathrm{H} 2$-type zinc finger transcription factor VuSTOP1 in rice bean (Vigna umbellata) reveals differential regulation between low pH and aluminum tolerance mechanisms. New Phytol. 208, 456-468. doi: 10.1111/nph.13456

Fujii, M., Yokosho, K., Yamaji, N., Saisho, D., Yanane, M., Takahashi, H., et al. (2012). Acquisition of aluminium tolerance by modification of a single gene in barley. Nat. Commun. 3:713. doi: 10.1038/ncomms1726

Furukawa, J., Yamaji, N., Wang, H., Mitani, N., Murata, Y., Sato, K., et al. (2007). An aluminum-activated citrate transporter in barley. Plant Cell Physiol. 48, 1081-1091. doi: 10.1093/pcp/pcm091

Gomez, C., Terrier, N., Torregrosa, L., Vialet, S., Fournier-Level, A., Verriès, C., et al. (2009). Grapevine MATE-type proteins act as vacuolar $\mathrm{H}+$-dependent acylated anthocyanin transporters. Plant Physiol. 150, 402-415. doi: $10.1104 /$ pp.109.135624

Green, L. S., and Rogers, E. E. (2004). FRD3 controls iron localization in Arabidopsis. Plant Physiol. 136, 2523-2531. doi: 10.1104/pp.104.045633

Higo, K., Ugawa, Y., Iwamoto, M., and Korenaga, T. (1999). Plant cis-acting regulatory DNA elements (PLACE) database: 1999. Nucleic Acids Res. 27, 297-300. doi: 10.1093/nar/27.1.297 
Jefferson, R. A., Kavanagh, T. A., and Benan, M. W. (1987). GUS fusions: $\beta$-glucuronidase as a sensitive and versatile gene fusion marker in higher plants. EMBO J. 6, 3901-3907.

Jones, D. L., Darah, P. R., and Kochian, L. V. (1996). Critical evaluation of organic acid mediated iron dissolution in the rhizosphere and its potential role in root iron uptake. Plant Soil. 180, 57-66. doi: 10.1007/BF00015411

Kobayashi, Y., Kobayashi, Y., Sugimoto, M., Lakshmanan, V., Iuchi, S., Kobayashi, M., et al. (2013). Characterization of the complex regulation of AtALMT1 expression in response to phytohormones and other inducers. Plant Physiol. 162, 732-740. doi: 10.1104/pp.113.218065

Lescot, M., Déhais, P., Thijs, G., Marchal, K., Moreau, Y., Van de Peer, Y., et al. (2002). PlantCARE, a database of plant cis-acting regulatory elements and a portal to tools for in silico analysis of promoter sequences. Nucleic Acids Res. 30, 325-327. doi: 10.1093/nar/30.1.325

Liu, M. Y., Chen, W. W., Xu, J. M., Fan, W., Yang, J. L., and Zheng, S. J. (2013). The role of VuMATE1 expression in aluminium-inducible citrate secretion in rice bean (Vigna umbellata) roots. J. Exp. Bot. 64, 1795-1804. doi: $10.1093 / \mathrm{jxb} / \mathrm{ert} 039$

Magalhaes, J. V., Liu, J., Guimarães, C. T., Lana, U. G., Alves, V. M., Wang, Y. H., et al. (2007). A member of the multidrug and toxic compound extrusion (MATE) family confers aluminum tolerance in sorghum. Nat. Genet. 39, 11561161. doi: $10.1038 / \mathrm{ng} 2074$

Ohwaki, Y., and Sugahara, K. (1997). Active extrusion of carboxylic acids in response to iron deficiency by roots of chickpea (Cicer arietinum L.). Plant Soil. 189, 49-55. doi: 10.1023/A:1004271108351

Omote, H., Hiasa, M., Matsumoto, T., Otsuka, M., and Moriyama, Y. (2006). The MATE proteins as functional transporters of metabolic and xenobiotic organic cations. Trends Pharmacol. Sci. 11, 587-593. doi: 10.1016/j.tips.2006.09.001

Porra, R., Thompson, W., and Kreidemann, P. (1989). Determination of accurate extinction coefficients and simultaneous equations for assaying chlorophylls a and $b$ extracted with four different solvents: verification of the concentration of chlorophyll standards by atomic absorption spectroscopy. Biochim. Biophys. Acta Bioenergetics 975, 348-394.

Rogers, E. E., and Guerinot, M. L. (2002). FRD3, a member of multidrug and toxin efflux family, controls iron deficiency responses in Arabidopsis. Plant Cell 14, 1787-1799. doi: 10.1105/tpc.001495

Ryan, P. R., and Delhaize, E. (2010). The convergent evolution of aluminium resistance in plants exploits a convenient currency. Funct. Plant Biol. 37, 275-284. doi: 10.1071/FP09261

Ryan, P. R., Raman, H., Gupta, S., Sasaki, T., Yamamoto, Y., and Delhaize, E. (2010). The multiple origins of aluminium resistance in hexaploid wheat include Aegilops tauschii and more recent cis mutations to TaALMT1. Plant J. 64, 446-455. doi: 10.1111/j.1365-313X.2010.04338.x

Sasaki, T., Ryan, P. R., Delhaize, E., Hebb, D. M., Ogihara, Y., Kawaura, K., et al. (2006). Sequence upstream of the wheat (Triticum aestivum L.) ALMT1 gene and its relationship to aluminum resistance. Plant Cell Physiol. 136, 4205-4214.

Serrano, M., Wang, B., Aryal, B., Garcion, C., Abou-Mansour, E., Heck, S., et al. (2013). Export of salicylic acid from the chloroplasts requires the multidrug and toxin extrusion-like transporter EDS5. Plant Physiol. 162, 1815-1821. doi: 10.1104/pp.113.218156

Shen, H., Ligaba, A., Yamaguchi, M., Osawa, H., Shibata, K., Yan, X., et al. (2004). Effect of K-252a and abscisic acid on the efflux of citrate from soybean roots. J. Exp. Bot. 55, 663-671. doi: 10.1093/jxb/erh058

Shoji, T., Inai, K., Yazaki, Y., Sato, Y., Takase, H., Shitan, N., et al. (2009). Multidrug and toxic compound extrusion-type transporters implicated in vacuolar sequestration of nicotine in tobacco roots. Plant Physiol. 149, 708-718. doi: $10.1104 /$ pp.108.132811

Sun, P., Tian, Q., Chen, J., and Zhang, W. H. (2010). Aluminium-induced inhibition of root elongation in Arabidopsis is mediated by ethylene and auxin. J. Exp. Bot. 61, 347-356. doi: 10.1093/jxb/erp306

Tian, Q., Zhang, X., Ramesh, S., Gilliham, M., Tyerman, S. D., and Zhang, W. H. (2014). Ethylene negatively regulates aluminium-induced malate efflux from wheat roots and tobacco cells transformed with TaALMT1. J. Exp. Bot. 65, 2415-2426. doi: 10.1093/jxb/eru123

Tokizawa, M., Kobayashi, Y., Saito, T., Kobayashi, M., Iuchi, S., Nomoto, M., et al. (2015). SENSITIVE TO PROTON RHIZOTOXICITY1, CALMODULIN BINDING TRANSCRIPTION ACTIVATOR2, and other transcription factors are involved in ALUMINUM-ACTIVATED MALATE TRANSPORTER1 expression. Plant Physiol. 167, 991-1003. doi: 10.1104/pp.114.256552

Tovkach, A., Ryan, P. R., Richardson, A. E., Lewis, D. C., Rathjen, T. M., Ramesh, S., et al. (2013). Transposon-mediated alteration of TaMATE1B expression in wheat confers constitutive citrate efflux from root. Plant Physiol. 161, 880-892. doi: 10.1104/pp.112.207142

Tsutsui, T., Yamaji, N., and Ma, J. F. (2011). Identification of a cis-acting element of ART1, a C2H2-type zinc-finger transcription factor for aluminum tolerance in rice. Plant Physiol. 156, 925-931. doi: 10.1104/pp.111.175802

von Bothmer, R., Sato, K., Komatsuda, T., Yasuda, S., and Fischbeck, G. (2003). "The demostication of cultivated barley," in Diversity in Barley (Hordeum vulgare), eds R. von Bothmer, Th. van Hintum, H. Knüpffer, and K. Sato (Amsterdam: Elservier Science), 9-27.

Wray, G. A., Hahn, M. W., Abouheif, E., Balhoff, J. P., Pizer, M., Rockman, M. V., et al. (2003). The evolution of transcriptional regulation in eukaryotes. Mol. Biol. Evol. 20, 1377-1419. doi: 10.1093/molbev/msg140

Yamaji, N., and Ma, J. F. (2007). Spatial distribution and temporal variation of the rice silicon transporter Lsil. Plant Physiol. 143, 1306-1313. doi: 10.1104/pp.106.093005

Yang, J. L., Chen, W. W., Chen, L. Q., Qin, C., Jin, C. W., Shi, Y. Z., et al. (2013). The 14-3-3 protein GENERAL REGULATORY FACTOR11 (GRF11) acts downstream of nitric oxide to regulate iron acquisition in Arabidopsis thaliana. New Phytol. 197, 815-824. doi: 10.1111/nph.12057

Yang, J. L., Zhang, L., Li, Y. Y., You, J. F., Wu, P., and Zheng, S. J. (2006). Citrate transporters play a critical role in aluminum-stimulated citrate efflux in rice bean (Vigna umbellata) roots. Ann. Bot. 97, 579-584. doi: 10.1093/aob/ mcl005

Yang, X. Y., Yang, J. L., Zhou, Y., Pineros, M. A., Kochian, L. V., Li, G. X., et al. (2011). A de novo synthesis citrate transporter, Vigna umbellate multidrug and toxic compound extrusion, implicates in Al-activated citrate efflux in rice bean (Vigna umbellata) root apex. Plant Cell Environ. 34, 2138-2148. doi: 10.1111/j.1365-3040.2011.02410.x

Yang, Z. B., Geng, X., He, C., Zhang, F., Wang, R., Horst, W. J., et al. (2014). TAA1regualted local auxin biosynthesis in the root-apex transition zone mediates the aluminum-induced inhibition of root growth in Arabidopsis. Plant Cell 26, 2889-2904. doi: 10.1105/tpc.114.127993

Yang, Z. M., Wang, J., Wang, S. H., and Xu, L. L. (2003). Salicylic acid-induced aluminum tolerance by modulation of citrate efflux from roots of Cassia tora $\mathrm{L}$. Planta 217, 168-174.

Yokosho, K., Yamaji, N., Ueno, D., Mitani, N., and Ma, J. F. (2009). OsFRDL1 is a citrate transporter required for efficient translocation of iron in rice. Plant Physiol. 149, 297-305. doi: 10.1104/pp.108.128132

Zhang, H., Zhu, H., Pan, Y., Luan, S., and Li, L. (2014). A DTX/MATEtype transporter facilitates abscisic acid efflux and modulates ABA sensitivity and drought tolerance in Arabidopsis. Mol. Plant 7, 1522-1532. doi: $10.1093 / \mathrm{mp} / \mathrm{ssu} 063$

Conflict of Interest Statement: The authors declare that the research was conducted in the absence of any commercial or financial relationships that could be construed as a potential conflict of interest.

Copyright (c) $2016 \mathrm{Liu}, \mathrm{Xu}, \mathrm{Lou}$, Fan, Yang and Zheng. This is an open-access article distributed under the terms of the Creative Commons Attribution License (CC BY). The use, distribution or reproduction in other forums is permitted, provided the original author(s) or licensor are credited and that the original publication in this journal is cited, in accordance with accepted academic practice. No use, distribution or reproduction is permitted which does not comply with these terms. 